3 Greenway D. Securing the Future of Excellent Patient Care: Final report of the independent review. Shape of Training, 2013.

Subodh Dave, Consultant Psychiatrist, Derbyshire Healthcare NHS Foundation Trust and Honorary Associate Professor, University of Nottingham, UK. Email: subodh.dave@derbyshcft.nhs.uk

doi: 10.1192/bjp.206.6.521a

Authors' reply: We broadly support Dr Dave's point concerning the need for closer working with primary care. Invariably, this will help prepare medical students for the reality of clinical practice post-qualification and additionally expose them to a less complex patient group. However we fall short of agreeing with the radical solution proposed.

The purpose of the editorial ${ }^{1}$ was to draw attention to the challenges faced by clinical psychiatry teachers in the context of the changing educational landscape and pressures. We have not suggested that the solutions proposed are sufficient alone. Rather, we contend that increasing transparency of funding, specifically developing learning opportunities in community teams and innovating undergraduate curricula, are especially timely and relevant given the ongoing challenges facing undergraduate psychiatry education delivery and its central importance as a 'shop window' for our profession.

Today's psychiatrists increasingly work in a supervisory capacity with team members, for example in community teams, and hence we propose there is an essential need to include undergraduate education within the design of new service delivery arrangements. Organisations with a focus on learning perform more effectively in terms of patient experience outcomes ${ }^{2}$ and, therefore, bringing undergraduates into our psychiatry team settings may ultimately benefit not only students, but also mental health trusts and patients. Creating an expansive learning culture with an emphasis on undergraduate teaching can only be positive.

With this in mind, we cannot support the radical solution proposed to transfer the majority of, if not all, undergraduate psychiatric education to primary care. Ultimately such a move would do medical students a disservice as they enter mental health rotations with a keenness to learn about psychiatry.

A primary-care-focused approach has the potential to undermine psychiatry as a profession by reinforcing stereotypes about the role of the psychiatrist being separate from medicine. Furthermore, undergraduate psychiatry attachments have been shown to be influential in improving medical students' attitudes and career intentions regarding psychiatry. ${ }^{3}$ It is unclear from the suggestion who would lead the teaching, how receptive primary care would be to such an imposition and importantly whether they have the skills or expertise to deliver our specialised outcomes as defined in the College's undergraduate curriculum. ${ }^{4}$

Rather than transfer psychiatry teaching to primary care, we advocate adopting a more integrated approach to teaching mental health, within both psychiatry rotations and the broader undergraduate medical curriculum. Integrated approaches have the additional benefit of aligning psychiatry teaching with mental health content that precedes or follows it. Importantly though, integration should not exclusively be with primary care. Joint teaching of delirium in hospital medicine rotations ${ }^{5}$ is another example of a focus of potential horizontal integration.

In summary, we thank Dr Dave for drawing attention to the importance of working closely with colleagues from other professions. However, before taking any radical steps there is an urgent need for improvements in the delivery of teaching in mental health settings and specifically in integrating psychiatry across the whole curriculum.
1 Abed R, Teodorczuk A. Danger ahead: challenges in undergraduate psychiatry teaching and implications for community psychiatry. $\mathrm{Br} \mathrm{J}$ Psychiatry 2015; 206: 89-90.

2 Berwick D. A Promise to Learn - A Commitment to Act. National Advisory Group on the Safety of Patients in England, 2013.

3 McParland M, Noble L, Livingston G, McManus, C. The effect of a psychiatric attachment on students' attitudes to and intention to pursue psychiatry as a career. Med Educ 2003; 37: 447-54.

4 Wilkinson P. Core Curriculum in Psychiatry. Royal College of Psychiatrists, 2011 (http://www.rcpsych.ac.uk/pdf/Undergraduate Psychiatry Curriculum 2011b.pdf).

5 Fisher JM, Gordon AL, MacLullich AM, Tullo E, Davis DH, Blundell A, et al. Towards an understanding of why undergraduate teaching about delirium does not guarantee gold-standard practice - results from a UK national survey. Age Ageing 2015; 44: 166-70.

Reem Abed, Consultant Psychiatrist, Northumberland, Tyne and Wear NHS

Foundation Trust, Andrew Teodorczuk, Consultant Psychiatrist and Honorary Senio Lecturer, School for Medical Education Development, Newcastle University, UK. Email: Andrew.Teodorczuk@ncl.ac.uk.

doi: 10.1192/bjp.206.6.522

\section{Comorbid medical illness in bipolar disorder}

Forty et $a l^{1}$ investigated whether the presence of physical comorbidity in individuals with bipolar disorder is associated with a more severe bipolar illness course that may contribute to the worsening of the mortality gap between individuals with bipolar disorder and the general community. Forty et al claimed this to be the first study on this issue in a UK clinical sample: that is, the first study that assessed rates of physical comorbidity in individuals with bipolar disorder and made direct comparisons with unipolar and control samples. The study is done statistically carefully.

While the results concerning the self-remembered physical comorbidities over the lifespan in the unipolar sample and the bipolar sample are clear, we have reservations concerning the composition of the control sample. Specifically, Forty et al used mixed samples of treatment-seeking individuals with unipolar and bipolar disorders that were originally recruited in genetic studies from psychiatric clinics, hospitals, general medical practices and self-help groups, as well as volunteers responding to media advertisements. ${ }^{2}$ The observed odds may be falsely calculated as the younger control sample is chosen, at least to a substantial proportion, from a specialised community sample that is representative neither of the general population nor of treatment-seeking individuals of the general community.

The reader might be interested to know that we recently published a paper on this issue. ${ }^{3}$ Between 1 January 2000 and 30 June 2012, 621 individuals with bipolar disorder were admitted to three general Manchester hospitals. All mental and physical comorbidities with a prevalence $>1 \%$ were compared with those of 6210 randomly selected and group-matched hospital controls of the same age and gender, regardless of priority of diagnoses. Comorbidities that increased the risk for hospital-based mortality (but not mortality outside of the hospitals) were identified using multivariate logistic regression analyses. The study was intended to determine which specific mental and physical comorbidities contribute to later in-hospital deaths in individuals with bipolar disorder, and whether the risk factors for hospital-based mortality differ for individuals with bipolar disorder in comparison with hospital controls.

In our view, our study has the advantage that a more representative and more relevant control sample was used and that the most relevant outcome from comorbidity - mortality - was addressed. In partial agreement with Forty et al, we found that excess comorbidity in individuals with bipolar disorder was caused by asthma and type 2 diabetes mellitus (T2DM). In addition, T2DM in individuals with bipolar disorder represented 
a major risk factor for general hospital-based mortality with excess mortality due to acute T2DM, as well as other diabetes-related complications. Our study gives support for an aggressive multidisciplinary approach to identify and treat T2DM to prevent diabetic, respiratory and vascular complications in all individuals with bipolar disorder.

1 Forty L, Ulanova A, Jones L, Jones I, Gordon-Smith K, Fraser C, et al. Comorbid medical illness in bipolar disorder. Br J Psychiatry 2014; 205 465-72.

2 Korszun A, Moskvina V, Brewster S, Craddock N, Ferrero F, Gill M, et al. Familiality of symptom dimensions in depression. Arch Gen Psychiatry 2004 61: 468-74.

3 Schoepf D, Heun R. Bipolar disorder and comorbidity: Increased prevalence and increased relevance of comorbidity for hospital-based mortality during a 12.5-year observation period in general hospital admissions. J Affective Disorders 2014; 169: 170-8.

Dieter Schoepf, Department of Psychiatry and Psychotherapy, University of Bonn Germany, Reinhard Heun, Department of Psychiatry, Radbourne Unit, Royal Derby Hospital, UK. Email: Dieter.Schoepf@ukb.uni-bonn.de

doi: $10.1192 /$ bjp.206.6.522a
Authors' reply: We agree with Schoepf \& Heun about the importance of control-sample characteristics in case-control comparisons and the need to consider the possibility of biased or unrepresentative findings. That is why, in our study, we used age as a covariate to take account of the younger age of controls compared with our mood disorder cases. We also discussed the potential limitations on generalisability caused by using a voluntary sample from a single ethnic group.

Schoepf \& Heun describe a different methodology (with its own strengths and limitations) to examine a different issue, namely the influence of comorbidity on mortality. It is encouraging that the findings from the two different approaches are consistent in highlighting the clinical importance of comorbidity of physical illness with bipolar disorder. Psychiatrists and mental health services must ensure that patients with bipolar disorder have access to appropriate, high-quality physical, as well as psychiatric, care.

Liz Forty, Nick Craddock (on behalf of the authors), Institute of Psychological Medicine and Clinical Neurosciences, Cardiff University, UK. Email: craddockn@cardiff.ac.uk

doi: $10.1192 /$ bjp.206.6.523 